\title{
Novel therapeutic trends in pneumonia: antibiotics and mes- enchymal stem cells
}

\author{
Jennifer Ly ${ }^{1}$, Qi Chu ${ }^{1}$, and Li Zhong ${ }^{1,2^{*}}$
}

1 Graduate College of Biomedical Sciences, Western University of Health Sciences, Pomona, California 91766, USA; jennifer.ly@westernu.edu (JL); qi.chu@westernu.edu (QC)

2 College of Osteopathic Medicine of the Pacific, Western University of Health Sciences, Pomona, California 91766, USA; lzhong@westernu.edu

* Correspondence: lzhong@westernu.edu; Tel.: 909-469-8220

\begin{abstract}
Pneumonia remains a major cause of morbidity and mortality worldwide, especially during COVID-19 pandemic. With the significant global health burden that pneumonia poses, it is essential to improve therapeutic and management strategies. The increasing emergence of antibiotic resistant bacterial strains limits options for effective antibiotic use. New antibiotics for treatment of pneumonia may address deficits in current antimicrobial drugs, with an ability to cover both typical, atypical, and resistance pathogen. Several of these newer drugs also have structural characteristics that allow for a decreased propensity in development of bacterial resistance. Potential use of stem cell therapies in place of corticosteroid treatments may also offer an improvement in patient outcomes. Human mesenchymal stem cell treatments have shown efficacy and safety in treating COVID-19 induced pneumonia. Combined treatment with both stem cells and antibiotics in pneumonia in a rabbit model has also shown significantly increased efficacy in comparison to antibiotic treatment alone, presenting yet another possible route for a novel strategy in treating pneumonia, though additional future studies are necessary before clinical implementation. While pneumonia remains a major disease of concern, having newer approved antibiotics as well as novel therapies such as stem cell treatments in the pipeline offers clinicians more options in effectively treating pneumonia.
\end{abstract}

Keywords: Community-Acquired Pneumonia; Hospital-Acquired Pneumonia; COVID-19; Antibiotics; Mesenchymal Stem Cells; Corticosteroids

\section{Introduction}

Pneumonia, defined as infection of the lung or pulmonary parenchyma by bacteria, viruses, fungi, and/or bacteria-like organisms, is one of the leading causes of morbidity and mortality globally [1-3]. Pneumonia is characterized by symptoms including fever, sweating, shortness of breath, chest pain, fatigue, and loss of appetite [1]. The most common pneumonia is community-acquired pneumonia (CAP), which is pneumonia acquired outside of a hospital setting [3]. S. pneumoniae, H. influenzae, S. aureus, and the influenza virus, are the main causative agents of CAP, with S. pneumoniae accounting for more than $25 \%$ of cases of CAP worldwide $[1,3,4]$. Hospital-acquired pneumonia (HAP), another common form of pneumonia, is acquired after at least 48 hours of hospitalization for other diseases $[1,3]$. While HAP can be caused by gram-positive cocci such as $S$. aureus and S. pneumoniae, gram-negative bacilli such as P. aeruginosa, K. pneumoniae, E. coli, and Enterobacter are more likely to be involved in HAP.

CAP has been shown to greatly increase the risk of long-term morbidity and mortality as well as the rates of all-cause hospitalization, emergency department visits, and CAP-related hospital visits [5]. In 2019, pneumonia and influenza were the ninth leading cause of death in the United States, accounting for over 49,000 deaths [6]. Pneumonia-related deaths in patients admitted to the intensive care unit (ICU) was approximately 30\%, however, with the COVID-19 pandemic caused by the novel Severe Acute 
Respiratory Syndrome Coronavirus 2 (SARS-CoV-2), mortality has increased to 35-50\% [7]. The COVID-19 pandemic has further highlighted the dangers of viral pneumonia, with over 2.7 million deaths worldwide and over 539,000 deaths in the United States as of March 24, $2021[8,9]$.

With the significant global health burden that pneumonia poses, it is essential to improve therapeutic and management strategies [10]. Although there are many treatments available, including antibiotics, corticosteroids, breathing treatments, and oxygen therapy, the increasing emergence of antibiotic resistant bacterial strains limits options for effective antibiotic use $[3,10,11]$. Furthermore, approximately $14-35 \%$ of hospitalized CAP patients die despite appropriate antibiotic treatments [12]. Beyond the problems associated with antibiotics, the use of corticosteroids has been controversial due to their associated adverse side effects, demonstrating the need for other therapeutic options. In this review, we summarize the most updated research and therapeutic guidelines for treating pneumonia, specifically antibiotics and mesenchymal stem cell therapies.

\section{Novel antibiotic treatments}

Although antibiotics remain the primary treatment for bacteria-induced pneumonia,, many of the current available pharmacological agents have limitations, including allergies, antibiotic resistance, inadequate penetration in lung tissues, and adverse side effects [13]. While most common CAP-causing Enterobacteriaceae are generally susceptible to typical antibiotics, a high prevalence of S. pneumoniae strains (20-40\%) are resistant to macrolides [14]. Current levels of resistance to fluroquinolones are still relatively low but increasing [14]. Furthermore, fluoroquinolones have been associated with adverse side effects, prompting the United States Food and Drug Administration to suggest that they be reserved for patients with no other treatment options [14, 15]. High rates of treatment failure caused by inadequate antibiotic treatments have led to increased mortality and morbidity as well as longer hospital stays, highlighting the need for newer effective antibiotics [11]. These new antibiotics may address deficits in current antimicrobial drugs, with an ability to cover both typical, atypical, and resistant pathogens $[7,16]$. While their use in severe CAP is not yet completely understood, these novel antibiotics may offer a potential treatment option for patients with resistant pathogens (Table 1).

\subsection{Communty-acquired pneumonia}

Lefamulin

Lefamulin is a pleuromutilin antibiotic that inhibits bacterial growth by binding to the peptidyl transferase center of the $50 \mathrm{~S}$ ribosome, preventing the binding of tRNA for peptide transfer and inhibiting peptide bond formation $[13,16,17]$. The binding pocket of the bacterial ribosome closes around the pleuromutilin, causing an induced fit and tightening the binding pocket $[13,17-19]$. This unique binding mechanism is believed to be the reason for the low potential for the development of bacterial resistance and cross-resistance to other antibiotics [13, 17-19]. Lefamulin exhibits both bactericidal and bacteriostatic activity against gram-positive, fastidious gram-negative, atypical pathogens, and some gram-negative anaerobes $[13,20]$. It has also been shown to achieve extensive penetration and accumulation in pulmonary epithelial lining fluid [13]. These properties suggest that lefamulin could target several limitations of current existing CAP therapies.

In a multicenter randomized control phase III trial (LEAP 1), IV-to-oral lefamulin was noninferior to IV-to-oral moxifloxacin in early clinical response $(87.3 \%$ vs. $90.2 \%$ respectively, difference $-2.9 \%, 95 \%$ CI -8.5 to 2.8 ) [21]. In a LEAP 2 trial, early clinical 
Table 1. Characteristics of Novel Antibiotics

\begin{tabular}{|c|c|c|c|c|}
\hline Drug Name & FDA Status & Antibiotic Class & Spectrum of Activity & Advantages \\
\hline Lefamulin & $\begin{array}{l}\text { Approval for CAP } \\
\text { treatment August } \\
2019\end{array}$ & Pleuromutilin & $\begin{array}{l}\text { Gram-positive strains (including } \\
\text { vancomycin-resistant enterococci and } \\
\text { MRSA), gram-negative strains, atypical } \\
\text { pathogens, pathogens typically associated } \\
\text { with CAP (i.e. S. pneumonia, H. influenzae) } \\
{[11,13,20]}\end{array}$ & $\begin{array}{l}\text { Unaffected by resistance to other antibiotic classes } \\
\text { including macrolides, fluoroquinolones, tetracy- } \\
\text { clines, b-lactam) } \\
\text { High specificity for inhibition of bacterial protein } \\
\text { translation with no effect on eukaryotic protein syn- } \\
\text { thesis, resulting in a low propensity for development } \\
\text { of bacterial resistance } \\
\text { Lack of cross-resistance with antibacterial classes }\end{array}$ \\
\hline Delafloxacin & $\begin{array}{l}\text { Approval for CAP } \\
\text { treatment } \\
\text { November } 2019\end{array}$ & Fluoroquinolone & $\begin{array}{l}\text { MRSA, penicillin-resistant/ } \\
\text { levofloxacin-resistant } S \text {. pneumoniae, } S \text {. } \\
\text { pyogenes, Enterococci, gram-negative } \\
\text { strains (including quinolone-susceptible } \\
\text { P. aeruginosa), atypical pathogens (C. } \\
\text { pneumoniae, M. pneumoniae, L. } \\
\text { pneumophila) }[11,15,19]\end{array}$ & $\begin{array}{l}\text { Penetrates well into ELF } \\
\text { More potent in vitro activity against most common } \\
\text { CAP pathogens } \\
\text { Lower mutant prevention concentrations compared } \\
\text { to other FQs }\end{array}$ \\
\hline Omadacycline & $\begin{array}{l}\text { Approval for CAP } \\
\text { treatment October } \\
2018\end{array}$ & Tetracycline & $\begin{array}{l}\text { MRSA, penicillin-resistant staphylococci, } \\
\text { gram-negative bacteria, atypical } \\
\text { pathogens }[11,22]\end{array}$ & $\begin{array}{l}\text { Active against } 2 \text { main forms of bacterial resistance to } \\
\text { tetracyclines: efflux and ribosomal protection } \\
\text { Has higher and sustained concentrations in plasma } \\
\text { and ELF }\end{array}$ \\
\hline Solithromycin & $\begin{array}{l}\text { Rejection in } 2017 \\
\text { (safety data required) }\end{array}$ & Macrolide & $\begin{array}{l}\text { MRSA, S. pnuemoniae (includes macrolid, } \\
\text { penicillin, and quinolone resistant), } H \text {. } \\
\text { influenzae, atypical pathogens }[11,23,24]\end{array}$ & $\begin{array}{l}\text { Has potent in vitro activity against fluroquinolone, } \\
\text { macrolide, and penicillin-resistant isolates of } S \text {. } \\
\text { pneumoniae }\end{array}$ \\
\hline $\begin{array}{l}\text { Imipenem/ } \\
\text { Cilastatin/ } \\
\text { Relebactam } \\
\text { (IMI-REL) }\end{array}$ & $\begin{array}{l}\text { Approval for } \\
\text { HAP/VAP treatment } \\
\text { on June } 2020\end{array}$ & $\begin{array}{l}\beta \text {-lactam } \\
\text { (carbapenem)/ } \\
\beta \text {-lactamase } \\
\text { inhibitor } \\
\text { combination }\end{array}$ & $\begin{array}{l}\text { Gram-negative strains (including } \\
\text { multidrug resistant } P \text {. aeruginosa, } \\
\text { carbapenum-resistant Enterobacterales, } \\
\text { extended-spectrum } \beta \text {-lactamases, } K \text {. } \\
\text { pneumonia) }[11,25-27]\end{array}$ & $\begin{array}{l}\text { Protection against several imipenem-resistant bacte- } \\
\text { ria }\end{array}$ \\
\hline
\end{tabular}


response rates were $90.8 \%$ for oral lefamulin and $90.8 \%$ for oral moxifloxacin (difference $0.1 \%$, 1 -sided $97.5 \% \mathrm{CI},-4.4 \%$ to $\infty$ ) [21]. The most frequently reported treatmentemergent adverse events were gastrointestinal, (diarrhea $12.2 \%$ in lefamulin, $1.1 \%$ in moxifloxacin; nausea $5.2 \%$ in lefamulin and $1.9 \%$ in moxifloxacin) [21]. Both studies showed that lefamulin was noninferior to moxifloxacin and was safe and well-tolerated [21].

Lefamulin has been shown to be an effective and well-tolerated agent, with availability in both oral and IV formulations to treat CAP. Patients who may benefit from lefamulin include those at higher risk of adverse events from fluoroquinolone use, those with a history of $C$. difficile infection, or those in settings with high prevalence of community-associated methicillin-resistant $S$. aureus (MRSA) [18].

\section{Delafloxacin}

Delafloxacin is an anionic fluoroquinolone with a unique structure that allows for increased intracellular penetration in bacteria, enhancing bactericidal activity in acidic conditions [28]. This property is a unique aspect of delafloxacin, as many other agents including other fluoroquinolones, macrolides, and aminoglycosides typically exhibit decreased antibacterial potency in acidic conditions [28]. Delafloxacin targets both topoisomerase IV and DNA gyrase to inhibit bacterial DNA replication [15, 28, 29]. This increased intracellular penetration in combination with delaxlfoxacin's unique mechanism give it a broad spectrum of activity against gram-positive, gram-negative, and atypical organisms, and is approved for treatment of CAP caused by S. pneumoniae, S. aureus (methicillin-susceptible isolates only), K. pneumoniae, E. coli, P. aeruginosa, $H$. influenzae, H. parainfluenzae, C. pneumoniae, L. pneumophila, and M. pneumoniae [28].

In a multicenter randomized phase III clinical trial (DEFINE-CABP), delafloxacin was shown to be noninferior to moxifloxacin $(88.9 \%$ vs. $89.0 \%$ respectively, difference $-0.2 \%, 95 \%$ CI $-4.4 \%$ to $4.1 \%$ ) [30]. Treatment-emergent adverse events occurred in $15.2 \%$ of the subjects in the delafloxacin group and $12.6 \%$ in the moxifloxacin group, with most events considered mild in severity [30]. Based on baseline MIC $_{90}$ values, delafloxacin demonstrated 16-fold greater activity compared to moxifloxacin for gram-positive and gram-negative pathogens [30].

Overall, delafloxacin has been shown to be noninferior to moxifloxacin and is effective and generally well-tolerated. In particular, it can be a potential treatment for patients with comorbidities, specifically COPD/asthma, based on improved response in these patients in the DEFINE-CABP trial [28]. Delafloxacin is a promising new antibiotic given its mild side effect profile, including a lack of association with QTc prolongation, a typical side effect of quinolones [15, 31].

\section{Omadacycline}

Omadacycline is an aminomethylcycline that was created via chemical modification of minocycline and was developed to target Tet(A) and other Tet efflux genes [22, 32]. The chemical modifications to its structure allow it to be active against two forms of bacterial resistance to tetracyclines: efflux and ribosomal protection. Omadacycline remains active and relatively unaffected by the presence of tetracycline efflux pumps (i.e. TetK) and ribosomal protection proteins (i.e. TetM), as well as other resistance mechanisms to other antibiotic classes [19]. It is active against staphylococci (including methicillin-resistant strains) and streptococci (including tetracycline-resistant strains). Additionally, omadacycline has a higher affinity for the 305 ribosomal subunit than tetracycline, binding to the $30 \mathrm{~S}$ ribosome to inhibit protein synthesis [19].

In a randomized phase III clinical trial (OPTIC), omadacycline was shown to be noninferior to moxifloxacin, with clinical success rates of $88.4 \%$ for omadacycline compared to $85.2 \%$ with moxifloxacin (difference $3.3 \%, 97.5 \%$ CI -2.7 to 9.3 ) [33]. Both treatments showed high rates of clinical success overall in patients with an identified 
CAP pathogen, as well as against gram-positive, gram-negative, and atypical pathogens [33]. Furthermore, there was no evidence of decreasing susceptibility to omadacycline during treatment. However, there is also a mortality imbalance in patients with CAP (2\% in the omadacycline group compared to $1 \%$ in moxifloxacin group) [33]. As the cause of the mortality imbalance has not yet been established, patients with CAP on omadacycline should be closely monitored [33]. The United States Food and Drug Administration (FDA) has required an additional active-controlled safety study in pediatric patients age 8-17 to further define omadacycline's safety and efficacy in treatment of CAP $[19,34]$.

In addition to its mild side effect profile, omadacycline can be used to treat patients with known hypersensitivity or intolerance to vancomycin and $\beta$-lactams, making it another potential option for treatment of CAP [22].

\section{Solithromycin}

Solithromycin is a "fourth generation" macrolide and fluoroketolide that has yet to receive FDA approval for treatment of CAP [16, 35]. Solithromycin binds the 50S ribosomal subunit near the peptide exit tunnel, prematurely terminating translation and causing frameshift errors in translation [24, 36]. This mechanism is considered bactericidal, however, due to its added ability to interfere with the formation of the ribosomal $50 \mathrm{~S}$ unit, ketolides are considered bacteriostatic [36]. Furthermore, ketolides have been found to be less sensitive to macrolide efflux (mef), contributing to solithromycin's restored activity against $H$. influenzae [36]]. Its structure helps solithromycin to overcome macrolide resistance in addition to problems with adverse events of telithromycin [36]. In particular, solithromycin lacks a pyridine moiety which may reduce hepatic toxicity, and has a fluorine at C-2, improving drug binding and enhancing activity [24, 36].

A multicenter double-blind randomized phase II study demonstrated that solithromycin has comparable efficacy and favorable safety compared to levofloxacin, with an $84.6 \%$ efficacy outcome rate of clinical success in the solithromycin group compared to $86.6 \%$ in the levofloxacin group, and early response success rates at $72.3 \%$ vs. $71.6 \%$ respectively [23]. Majority of treatment-emergent adverse events were mild-to-moderate gastrointestinal symptoms (diarrhea $7.8 \%$ in solithryomycin vs. 5.9\% in levofloxacin; nausea $1.6 \%$ in solithromycin vs. $10.3 \%$ in levofloxacin; vomiting $0 \%$ in solithromycin vs. $4.4 \%$ in levofloxacin) [23].

Subsequent phase III trials used moxifloxacin as the comparator. A multicenter double-blind randomized phase III trial (SOLITAIRE-ORAL) compared the efficacy and safety of oral solithromycin with oral moxifloxacin and showed that solithromycin was noninferior to moxifloxacin in treatment of CAP [37]. Early clinical response was achieved in $78.2 \%$ of the solithromycin group compared to the $77.9 \%$ in the moxifloxacin group (difference $0.29 \%, 95 \%$ CI -5.5 to 6.1) [37]. Both drugs showed similar safety profiles, with a $10 \%$ incidence of treatment-emergent adverse events in the solithromycin group compared to the $13 \%$ in the moxifloxacin group [37]. The most common adverse events were mild gastrointestinal symptoms (diarrhea $4 \%$ in solithromycin vs. $6 \%$ in moxifloxacin; nausea $4 \%$ in both groups; vomiting $2 \%$ in both groups) and nervous system symptoms (headache $4 \%$ in solithromycin vs. $3 \%$ in moxifloxacin; dizziness $2 \%$ in both groups) [37]. It was noted that overall ALT concentrations of $>3$ times, $>5$ times, and $>10$ times the upper limit of normal were noted in $7.2 \%, 2.4 \%$, and $0.1 \%$ of patients in the solithromycin group in comparison to the $3.6 \%, 1.0 \%$, and $0.2 \%$ in the moxifloxacin group $[24,37]$

A second phase III trial (SOLITAIRE-IV) evaluated the safety and efficacy of IV-to-oral solithromycin and moxifloxacin, also supporting prior studies' conclusions that solithromycin was noninferior to moxifloxacin [38]. Early clinical response was achieved in $79.3 \%$ of the solithromycin group compared to the $79.7 \%$ in the moxifloxacin 
group (difference $-0.46 \%, 95 \%$ CI -6.1 to 5.2) [38]. Adverse events were comparable between the two groups, though mostly mild/moderate infusion events led to a higher incidence of adverse events in the solithromycin group [38].

Solithromycin is an effective antibiotic regiment that offers additional advantages, including its anti-inflammatory effect and its potent activity against pathogens [35]. However, due to concerns related to hepatoxicity, the FDA has recommended further clinical studies to assess the safety profile in 9000 patients [16, 24].

\subsection{Hospital-acquired pneumonia}

Imipenem/Cilastatin/Relebactam (IMI-REL)

IMI-REL is a new intravenously-administered $\beta$-lactam (carbapenem)/ $\beta$-lactamase inhibitor anti-infective combination antibiotic that recently received FDA approval for use in treating HAP and ventilator-associated pneumonia (VAP) in June 2020 [26]. Imipenem is a carbapenem that inactivates penicillin-binding proteins to inhibit peptidoglycan crosslinking during cell wall synthesis, resulting in bacterial cell lysis and death [26]. It is coadministered with cilastatin, a dehydropeptidase-I inhibitor that does not have antibacterial activity and simply reduces renal metabolism $[25,26]$. Relebactam is a novel $\beta$-lactamase inhibitor that protects imipenem from degradation by Pseudomonas-derived cephalosporinase and class A and C $\beta$-lactamases, helping to restore imipenem activity against several imipenem-resistant bacteria including $P$. aeruginosa and Enerobacteriaceae [25-27]. IMI-REL has a broad spectrum range in vitro, including multidrug resistant $P$. aeruginosa and carbapenum-resistant Enterobacterales [25-27].

Two phase III trials of IMI-REL have been conducted to study its efficacy and safety. In the double-blind randomized phase III trial (RESTORE-IMI 1), the efficacy and safety of IMI-REL was comparable to that of imipenem/cilastin + colistin for the treatment of hospitalized patients with hospital-acquired/ventilator-associated pneumonia, complicated intraabdominal infection, or complicated urinary tract infection caused by imipenem-nonsusceptible pathogens [39]. Favorable overall response was observed in $71 \%$ of the IMI-REL group compared to $70 \%$ of the imipenem + colistin group. Serious adverse events occurred in $10 \%$ of the IMI-REL group as opposed to $31 \%$ in the imipenem + colistin group [39]. The second double-blind randomized control phase III trial (RESTORE-IM 2) demonstrated noninferiority of IMI-REL compared to piperacillin/ tazobactum in patients with HAP/VAP [40]. The favorable early clinical response was $61.0 \%$ in the IMI-REL group compared to the $55.8 \%$ in the piperacillin/tazobactam group; Day 28 all-cause mortality was $15.9 \%$ in the IMI-REL group compared to $21.3 \%$ in the piperacillin/tazobactam group (difference $-5.3 \%, 95 \%$ CI -3.2 to 13.2) [40]. Serious adverse events occurred in $26.7 \%$ of the IMI-REL group vs. $32.0 \%$ of the piperacillin/tazobactam group [40]. Common adverse events included anemia, elevated liver enzymes, gastrointestinal symptoms (nausea, vomiting, diarrhea), and headaches [40].

The two studies demonstrate that IMI-REL is generally well-tolerated and is a viable treatment option for gram-negative HAP/VAP, including in critically ill, high-risk patients.

\section{Corticosteroids}

The official clinical practice guideline of the American Thoracic Society (ATS) and Infectious Diseases Society of America (IDTA) for the diagnosis and treatment of CAP recommends corticosteroid treatment only for patients with CAP and refractory septic shock [41]. The use of corticosteroids in decreasing inflammation patients with severe CAP has been studied for decades with conflicting results [7, 12]. Corticosteroids can be used as adjuvant therapy for acute respiratory distress syndrome, though use in severe viral pneumonia is controversial due to numerous negative side effects associated with it [42]. 
Potentially, corticosteroids may decrease cytokines to reduce inflammation and help with inadequate adrenal response in critically ill patients [43]. Because antibiotics are largely used to treat bacteria-induced pneumonia, steroids are mainly tested for treatment of viral-induced pneumonia. Due to the controversies surrounding corticosteroid use in treating CAP, many clinical trials have been performed to evaluate the safety and efficacy of several steroids (Table 2).

In light of the COVID-19 pandemic, several clinical trials have been conducted to determine the efficacy and safety of steroids in treating SARS-CoV-2 induced pneumonia. In a multicenter randomized control study done by Abd-Elsalam et al. evaluating the safety and efficacy of hydroxychloroquine (HCQ) with standard care in patients with COVID-19, the overall mortality did not differ between the control and experimental group $(6.2 \%$ patients with HCQ + standard care vs. $5.2 \%$ patients with standard care alone) [44]. Moreover, patients in the HCQ + standard care group experienced more negatives side effects (i.e. difficulty seeing and hearing, unusual bleeding, irregular heartbeat, etc.) compared to the control group [44].

Dequin et al. conducted a clinical trial on the efficacy of low-dose hydrocortisone in patients with COVID-19 induced acute respiratory failure and found that there was no significant difference in rate of death or persistent respiratory support with mechanical ventilation or high-flow oxygen therapy between the group treated with low-dose hydrocortisone and the control group (42.1\% in the treatment vs 50.7\% in the control) [45].

Tomazini et al. conducted a multicenter randomized open-label clinical trial in 41 ICUs in Brazil to determine if intravenous dexamethasone could treat SAR-CoV-2 induced acute respiratory distress syndrome (ARDS) [46]. Sequential Organ Failure Assessment (SOFA) scores (range, 0-24, with higher scores indicating greater organ dysfunction) were used to evaluate the differences [46]. At 7 days, patients in the dexamethasone treatment group had a mean SOFA score of 6.1 (95\% CI, 5.5 to 6.7) in comparison to a $7.5(95 \% \mathrm{CI}, 6.9$ to 8.1$)$ in the standard care group (difference, $-1.16 ; 95 \% \mathrm{CI},-1.94$ to $-0.38 ; \mathrm{P}=.004$ ) [46]. However, at day $28,21.9 \%$ in the dexamethasone group compared to the $29.1 \%$ in the standard care group experienced secondary infections, and $3.3 \%$ vs $6.1 \%$ experienced serious adverse events [46]. This study showed that dexamethasone treatment decreases secondary infections and serious adverse events [46]. Despite its efficacy, however, it is important to note that dexamethasone is associated many side effects, including mental depression, mood change, headache, and irregular heartbeat.

Lastly, Edalatifard et al. conducted a single-blind randomized controlled clinical trial in Iran to evaluate methylprednisolone's ability to reduce the inflammation of respiratory system in patients with COVID-19 [47]. There was a higher incidence of improvement in the methylprednisolone treatment group than in the standard care group $(94.1 \%$ vs. $57.1 \%)$ [47]. Furthermore, the mortality rate was significantly lower in the methylprednisolone group $(5.9 \%$ vs. $42.9 \%$; $<<0.001)$ [47]. The results of this study suggest that that methylprednisolone could be an efficient therapeutic agent [47].

Based on the results of the multiple clinical trials conducted, most steroids were not very effective in treating pneumonia. Although some steroids may have shown efficacy in treating pneumonia, they are also associated with many negative adverse events, prompting the ATS/IDTA to recommend against routine corticosteroid use in treating CAP and severe influenza pneumonia [41]. In view of these adverse events and recommendations, several studies have been conducted evaluating the use of alternative therapies in place of corticosteroids. 
Table 2. Comparison of Clinical Trials Studying Treatment of Pneumonia with Corticosteroids

Abd-Elsalam et al. [44], 2020

Dequin et al. [45], 2020

Tomazini et al. [46], 2020

Edalatifard et al. [47], 2020

\begin{tabular}{|c|c|c|c|c|}
\hline Patients & 194 & 149 & 299 & 68 \\
\hline Study Design & $\begin{array}{c}\text { Multicenter, } \\
\text { randomized control }\end{array}$ & $\begin{array}{l}\text { Multicenter randomized } \\
\text { double-blind sequential }\end{array}$ & $\begin{array}{c}\text { Multicenter, randomized, } \\
\text { open-label, clinical }\end{array}$ & $\begin{array}{c}\text { Single-blind, randomized } \\
\text { control clinical }\end{array}$ \\
\hline Age, yr (mean \pm SD) & $40.72 \pm 19.32$ & 62.2 & $61 \pm 14$ & $58.5 \pm 16.6$ \\
\hline Steroid & Hydroxychloroquine & Hydrocortisone & Dexamethasone & Methylprednisolone \\
\hline Outcomes & Mortality & Rate of treatment failure & Ventilator-free days & Condition improvement \\
\hline Treatment Group & $6.2 \%$ & $42.1 \%$ & $6.6 \%$ & $94.1 \%$ \\
\hline Control Group & $5.2 \%$ & $50.7 \%$ & $4.0 \%$ & $57.1 \%$ \\
\hline Adverse Events & NR & $\begin{array}{c}\text { No serious adverse events } \\
\text { attributed }\end{array}$ & $\begin{array}{l}\text { Acute MI, DVT, GI } \\
\text { perforation, unspecified } \\
\text { hyperglycemia, } \\
\text { pneumothorax }\end{array}$ & $\begin{array}{c}\text { Fever, dyspnea, GI symptoms } \\
\text { myalgia, headache, cough, } \\
\text { weakenss }\end{array}$ \\
\hline Treatment Group & NR & $0 \%$ & $3.3 \%$ & $5.8 \%$ \\
\hline Control Group & NR & $0 \%$ & $6.1 \%$ & $7.1 \%$ \\
\hline
\end{tabular}

* Defined as death or persistent respiratory support with mechanical ventilation or high-flow oxygen therapy

NR: not reported; MI: myocardial infarction; GI: gastrointestinal 


\section{Mesenchymal stem cell treatments}

Mesenchymal stem cells (MSCs) are adult multipotent stem cells found in bone marrow that have potent immunomodulatory properties to suppress the pro-inflammatory processes in the lungs. MSC treatments may have a specific therapeutic potential and safety profile in treatment of pneumonia that may be used in place of corticosteroid therapies [48].

\subsection{MSCs show both steroid-like and antibiotic effects on bacteria-induced pneumonia}

The first line of treatment for bacteria-induced pneumonia is empiric prescription of antibiotics, while stem cell therapies are being considered as a replacement for corticosteroid treatments. Gupta et al. conducted a study using a mouse model of gram-negative pneumonia to evaluate the efficacy of MSCs [49]. MSCs could reduce lung injury and increase survival (55\% in the MSC group vs. $8 \%$ in the $3 \mathrm{~T} 3$ control and $0 \%$ in the PBS control group). Furthermore, MSCs were noted to enhance bacterial clearance in the alveolar space as early as 4 hours following administration and was still able to retain their classic immunosuppressive properties [49]. Moreover, MSCs significantly upregulated their production of lipocalin 2, an innate immune protein used to prevent growth and spread of microorganisms $[49,50]$. The same research group also conducted a study to test the effect of human MSCs derived from bone marrow on the bacterial growth of gram-negative (E. coli and P. aeruginosa) and gram-positive (S. aureus) bacteria, and found that MSCs directly inhibited bacterial growth [50]. While Gupta et. al concluded that MSCs could be beneficial in bacteria-induced pneumonia, it has not yet been clinically tested in human. Clinical trials would be required to determine the efficacy and safety of MSCs in treating bacteria-induced pneumonia in humans.

\subsection{MSCs show efficacy and safety in virus-induced pneumonia}

A majority of the studies evaluating the efficacy and safety of MSCs have been performed on virus-induced pneumonia. While the efficacy and safety of MSCs in treating bacteria-induced pneumonia has not yet been determined in humans, there have been numerous clinical trials performed to test the safety and efficacy of MSCs in treating virus-induced pneumonia (Table 3).

Meng et al. conducted a parallel non-randomized phase I clinical trial evaluating the safety of human umbilical cord-derived mesenchymal stem cell (UC-MSCs) infusions on patients with moderate-to-severe COVID-19 pulmonary disease [51]. No serious UC-MSCs infusion-associated adverse events were observed and all patients had recovered and were discharged, demonstrating that intravenous UC-MSCs infusions are safe [51].

Two individual clinical studies were conducted testing both the efficacy and the safety of UC-MSCs in treating COVID-19-induced pneumonia. Shi et al. demonstrated that the administration of UC-MSCs improved whole lung lesion volume from baseline to day 28 compared to the placebo group (the median difference $-13.31 \%, 95 \%$ CI $-29.14 \%$ to $2.13 \%, \mathrm{P}=0.080$ ) [52]. UC-MSCs also significantly reduced the proportions of solid component lesion volume (median difference $-15.45 \%$; $95 \%$ CI $-30.82 \%$ to $0.39 \%$; $\mathrm{P}=$ 0.043). Moreover, the incidence of adverse events was similar between the treatment group and the placebo group, indicating that UC-MSCs were not only effective, but also did not have severe side effects and were safe to use [52]. Similarly, Shu et al. found that patients in the UC-MSC had a shorter time to clinical improvement in comparison with the control group (median 9.0 days vs. 14.0 days respectively, $\mathrm{P}=0.006$ ) [53]. Furthermore, all of the patients in the treatment group had no adverse reactions (rash, allergic reaction, and febrile reaction) [53]. Both studies done by Shi et al. and Shu et al. demonstrated that the administration of UC-MSCs in treatment of COVID-19 induced pneumonia was both effective and safe. 
Biomedicines 2021, 9, x FOR PEER REVIEW

Table 3. Comparison of Clinical Trials Studying Treatment of Pneumonia with Mesenchymal Stem Cells

\begin{tabular}{|c|c|c|c|c|}
\hline & Meng et al. [51], 2020 & Shi et al. [52], 2021 & Shu et al. [53], 2020 & Hashemian et al. [54], 2021 \\
\hline Patients & 18 & 100 & 41 & 11 \\
\hline Study Design & $\begin{array}{c}\text { Parallel, non-randomized, } \\
\text { phase } 1 \text { control }\end{array}$ & $\begin{array}{l}\text { Phase II randomized, } \\
\text { double-blind, placebo-control }\end{array}$ & $\begin{array}{l}\text { Single-center open-label, } \\
\text { individually randomized, } \\
\text { standard treatment-control }\end{array}$ & $\begin{array}{l}\text { Phase I, two-center, } \\
\text { open-label, single-arm }\end{array}$ \\
\hline Age, yr (mean \pm SD) & NR & $\begin{array}{l}\text { Treatment: } 60.72 \pm 9.14 \\
\text { Control: } 59.94 \pm 7.79\end{array}$ & $58.78 \pm 16.26$ & $53.9 \pm 10.37$ \\
\hline Outcome & Efficacy not evaluated & $\begin{array}{c}\text { Change in whole lung lesion } \\
\text { volume }\end{array}$ & $\begin{array}{l}\text { Time to clinical } \\
\text { improvement* }\end{array}$ & Survival rate \\
\hline Treatment Group & $\mathrm{N} / \mathrm{A}$ & $-19.4 \%$ & $91.67 \%$ & $6 / 11$ \\
\hline Control Group & $\mathrm{N} / \mathrm{A}$ & $-7.30 \%$ & $51.72 \%$ & None \\
\hline Adverse Events & $\begin{array}{c}\text { No serious adverse events } \\
\text { attributed }\end{array}$ & $\begin{array}{c}\text { Increased LDH, increased } \\
\text { ALT, hypokalaemia, } \\
\text { increased AST } \\
\end{array}$ & $\begin{array}{c}\text { No serious adverse events } \\
\text { attributed }\end{array}$ & NR \\
\hline Treatment Group & $0 \%$ & $55.3 \%$ & $0 \%$ & NR \\
\hline Control Group & $0 \%$ & $60.0 \%$ & $0 \%$ & NR \\
\hline
\end{tabular}

* Clinical improvement was defined as a decline of two categories on the modified seven-category ordinal scale of clinical status, or hospital discharge 
Hashemian et al. also conducted a clinical trial to evaluate the safety, feasibility, and tolerability of the multiple infusions of high dose MSCs derived from the placenta and umbilical cord in treating COVID-19 induced pneumonia [54]. No serious adverse events were reported 24-48 hours after cell infusions, and reduced dyspnea and increased oxygen saturation (SpO2) levels were observed within 48-96 hours after the first infusion in 7 out of 11 patients [54]. Overall, six patients survived with significant reductions in serum levels of tumor necrosis factor-alpha (TNF- $\alpha$; P $<0.01)$, IL-8 $(\mathrm{P}<0.05)$, and C-reactive protein $(\mathrm{CRP})(\mathrm{P}<0.01)$ [54]. Moreover, none of these patients had complaints of dyspnea on day 60 post-infusion, and lung computed tomography (CT) scans showed remarkable signs of recovery from COVID-19 [54]. Hashemian et al. concluded that multiple infusions of high- dose allogeneic prenatal MSCs were safe and effective [54].

In summary, MSC treatments are safe and effective in treating COVID-19 induced pneumonia, making them an ideal potential replacement for corticosteroid treatments for COVID-19 patients. Recently, the FDA has given conditional approval for use of stem cell treatments on severe COVID-19 patients under "expanded access compassionate use" $[51,52]$. However, further clinical trials may be necessary to further the establish efficacy and safety of MSC treatments, not only in treating COVID-19 patients, but also in treating other virus-induced pneumonias, especially if they are to be considered a replacement for corticosteroids.

\section{Combined stem cell and antibiotic treatments}

Because both MSCs and novel antibiotics have been shown to be promising therapies for the treatment of pneumonia respectively, a few studies have conducted to determine the efficacy and safety of combined stem cell and antibiotic treatments.

Kong et al. conducted a study testing the combined treatment of antibiotic linezolid and human MSCs (hUMSCs) on a rabbit model with MRSA-infected pneumonia [55]. Linezolid monotherapy ( $50 \mathrm{mg} / \mathrm{kg}$ for two times/day) resulted in improvement of body weight, chest imaging, bronchoscopic manifestations, histological parameters, and IL-10 concentration in plasma $(\mathrm{P}<0.01)$, decreasing pulmonary auscultation, and reduction of IL-8, IL-6, CRP, and TNF- $\alpha$ concentrations in plasma $(\mathrm{P}<0.01)$ when compared with the pneumonia model group at 48 and 168 hrs [55]. Coadministration of linezolid and hUMSCs $\left(1 \times 10^{6} / \mathrm{kg}\right.$ for two times at 6 and $72 \mathrm{hrs}$ after MRSA instillation) and further increased the body weight $(\mathrm{P}<0.05)$ and significant reduction of lung inflammation on $\mathrm{CT}$ scans [55]. Combination treatment with both stem cells and linezolid showed significant improved therapeutic effects in comparison to linezolid treatment alone [55]. Further clinical trials, however, are needed to show that this combination treatment is also effective and safe in treating pneumonia in humans.

\section{Conclusions}

Pneumonia continues to remain an important infection due to its impact on patient outcomes, especially amongst young children, the elderly, and immunocompromised patients. The availability of new antibiotics offers an opportunity for treatment of antibiotic-resistant pathogens associated with both CAP and HAP. These new drugs in particular have a broad spectrum of activity against pathogens, including multidrug resistant strains that pose a major threat to clinical practice given the limited therapeutic options. Moreover, in addition to having similar safety and efficacy profiles as older drugs, several of these newer drugs have structural characteristics that allow for a decreased propensity in the development of bacterial resistance.

In addition to novel antibiotics, potential use of stem cell therapies in place of corticosteroid treatments may offer an improvement in patient outcomes. Novel stem cell therapies, especially human UC-MSCs, showed efficacy and safety on COVID-19 induced pneumonia. However, further research and clinical trials are needed to demon- 
strate the efficacy and safety of UC-MSCs in treatment of other virus-induced pneumonias beyond COVID-19.

The combined treatment of both stem cells and antibiotics in pneumonia in a rabbit model showed significantly increased efficacy in comparison to antibiotic treatment alone, presenting a possible route for a novel strategy in treating pneumonia, though additional future studies are necessary before clinical implementation.

While pneumonia remains a major disease of concern, having newer approved antibiotics as well as novel therapies such as stem cell treatments in the pipeline offers clinicians more options in effectively treating pneumonia.

Author Contributions: Conceptualization, L.Z.; writing - original draft preparation, L.J. and Q.C.; writing - review and editing, L.Z.

All authors have read and agreed to the published version of the manuscript.

Funding: This research received no external funding.

Institutional Review Board Statement: Not applicable.

Informed Consent Statement: Not applicable.

Data Availability Statement: Not applicable.

Conflicts of Interest: The authors declare no conflict of interest.

\section{References}

1. Lanks, C.W., A.I. Musani, and D.W. Hsia, Community-acquired Pneumonia and Hospital-acquired Pneumonia. Med Clin North Am, 2019. 103(3): p. 487-501. doi: 10.1016/j.mcna.2018.12.008

2. Mandell, L.A., Community-acquired pneumonia: An overview. Postgrad Med, 2015. 127(6): p. 607-15. doi: 10.1080/00325481.2015.1074030

3. Kim, G.L., S.H. Seon, and D.K. Rhee, Pneumonia and Streptococcus pneumoniae vaccine. Arch Pharm Res, 2017. 40(8): p. 885-893. doi: 10.1007/s12272-017-0933-y

4. Musher, D.M. and A.R. Thorner, Community-acquired pneumonia. N Engl J Med, 2014. 371(17): p. 1619-28. doi: 10.1056/NEJMra1312885

5. $\quad$ Eurich, D.T., T.J. Marrie, J.K. Minhas-Sandhu, and S.R. Majumdar, Ten-Year Mortality after Community-acquired Pneumonia. A Prospective Cohort. Am J Respir Crit Care Med, 2015. 192(5): p. 597-604. doi: 10.1164/rccm.201501-0140OC

6. Kochanek, K.D., J. Xu, and E. Arias, Mortality in the United States, 2019. NCHS Data Brief, 2020(395): p. 1-8. doi:

7. Nair, G.B. and M.S. Niederman, Updates on community acquired pneumonia management in the ICU. Pharmacol Ther, 2021. 217: p. 107663. doi: 10.1016/j.pharmthera.2020.107663

8. Dong, E., H. Du, and L. Gardner, An interactive web-based dashboard to track COVID-19 in real time. Lancet Infect Dis, 2020. 20(5): p. 533-534. doi: 10.1016/S1473-3099(20)30120-1

9. WHO COVID-19 Dashboard. Geneva: World Health Organization, 2020. Available online: https://covid19.who.int.

10. Peyrani, P., L. Mandell, A. Torres, and G.S. Tillotson, The burden of community-acquired bacterial pneumonia in the era of antibiotic resistance. Expert Rev Respir Med, 2019. 13(2): p. 139-152. doi:

10.1080/17476348.2019.1562339

11. Russo, A., Spotlight on New Antibiotics for the Treatment of Pneumonia. Clin Med Insights Circ Respir Pulm Med, 2020. 14: p. 1179548420982786. doi: 10.1177/1179548420982786 antibiotics. Eur Respir Rev, 2015. 24(137): p. 516-24. doi: 10.1183/16000617.0034-2015 
13. Eraikhuemen, N., D. Julien, A. Kelly, T. Lindsay, and D. Lazaridis, Treatment of Community-Acquired Pneumonia: A Focus on Lefamulin. Infect Dis Ther, 2021. doi: 10.1007/s40121-020-00378-3

14. Falco, V., J. Burgos, and B. Almirante, An overview of lefamulin for the treatment of community acquired bacterial pneumonia. Expert Opin Pharmacother, 2020. 21(6): p. 629-636. doi: 10.1080/14656566.2020.1714592

15. Mogle, B.T., J.M. Steele, S.J. Thomas, K.H. Bohan, and W.D. Kufel, Clinical review of delafloxacin: a novel anionic fluoroquinolone. J Antimicrob Chemother, 2018. 73(6): p. 1439-1451. doi: 10.1093/jac/dkx543

16. Kollef, M.H. and K.D. Betthauser, New antibiotics for community-acquired pneumonia. Curr Opin Infect Dis, 2019. 32(2): p. 169-175. doi: 10.1097/QCO.0000000000000526

17. Watkins, R.R. and T.M. File, Lefamulin: A Novel Semisynthetic Pleuromutilin Antibiotic for Community-acquired Bacterial Pneumonia. Clin Infect Dis, 2020. 71(10): p. 2757-2762. doi: 10.1093/cid/ciaa336

18. Mercuro, N.J. and M.P. Veve, Clinical Utility of Lefamulin: If Not Now, When? Curr Infect Dis Rep, 2020. 22(9): p. 25. doi: 10.1007/s11908-020-00732-z

19. Zhanel, G.G., J. Esquivel, S. Zelenitsky, C.K. Lawrence, H.J. Adam, A. Golden, R. Hink, L. Berry, F. Schweizer, M.A. Zhanel, et al., Omadacycline: A Novel Oral and Intravenous Aminomethylcycline Antibiotic Agent. Drugs, 2020. 80(3): p. 285-313. doi: 10.1007/s40265-020-01257-4

20. Paukner, S., S.P. Gelone, S.J.R. Arends, R.K. Flamm, and H.S. Sader, Antibacterial Activity of Lefamulin against Pathogens Most Commonly Causing Community-Acquired Bacterial Pneumonia: SENTRY Antimicrobial Surveillance Program (2015-2016). Antimicrob Agents Chemother, 2019. 63(4). doi: 10.1128/AAC.02161-18

21. File, T.M., L. Goldberg, A. Das, C. Sweeney, J. Saviski, S.P. Gelone, E. Seltzer, S. Paukner, W.W. Wicha, G.H. Talbot, et al., Efficacy and Safety of Intravenous-to-oral Lefamulin, a Pleuromutilin Antibiotic, for the Treatment of Community-acquired Bacterial Pneumonia: The Phase III Lefamulin Evaluation Against Pneumonia (LEAP 1) Trial. Clin Infect Dis, 2019. 69(11): p. 1856-1867. doi: 10.1093/cid/ciz090

22. Dougherty, J.A., A.J. Sucher, E.B. Chahine, and K.C. Shihadeh, Omadacycline: A New Tetracycline Antibiotic. Ann Pharmacother, 2019. 53(5): p. 486-500. doi: 10.1177/1060028018818094

23. Oldach, D., K. Clark, J. Schranz, A. Das, J.C. Craft, D. Scott, B.D. Jamieson, and P. Fernandes, Randomized, double-blind, multicenter phase 2 study comparing the efficacy and safety of oral solithromycin (CEM-101) to those of oral levofloxacin in the treatment of patients with community-acquired bacterial pneumonia. Antimicrob Agents Chemother, 2013. 57(6): p. 2526-34. doi: 10.1128/AAC.00197-13 
30. Horcajada, J.P., R.A. Salata, R. Alvarez-Sala, F.M. Nitu, L. Lawrence, M. Quintas, C.Y. Cheng, S. Cammarata, and D.-C.S. Group, A Phase 3 Study to Compare Delafloxacin With Moxifloxacin for the Treatment of Adults With Community-Acquired Bacterial Pneumonia (DEFINE-CABP). Open Forum Infect Dis, 2020. 7(1): p. ofz514. doi: 10.1093/ofid/ofz514

31. Shiu, J., G. Ting, and T.K. Kiang, Clinical Pharmacokinetics and Pharmacodynamics of Delafloxacin. Eur J Drug Metab Pharmacokinet, 2019. 44(3): p. 305-317. doi: 10.1007/s13318-018-0520-8

32. Markham, A. and S.J. Keam, Omadacycline: First Global Approval. Drugs, 2018. 78(18): p. 1931-1937. doi: 10.1007/s40265-018-1015-2

33. Torres, A., L. Garrity-Ryan, C. Kirsch, J.N. Steenbergen, P.B. Eckburg, A.F. Das, M. Curran, A. Manley, E. Tzanis, and P.C. McGovern, Omadacycline vs moxifloxacin in adults with community-acquired bacterial pneumonia. Int J Infect Dis, 2021. 104: p. 501-509. doi: 10.1016/j.ijid.2021.01.032

34. Burgos, R.M. and K.A. Rodvold, Omadacycline: a novel aminomethylcycline. Infect Drug Resist, 2019. 12: p. 1895-1915. doi: 10.2147/IDR.S171352

35. Wen, J., F. Chen, M. Zhao, and X. Wang, Solithromycin monotherapy for treatment of community-acquired bacterial pneumonia: A meta-analysis of randomised controlled trials. Int J Clin Pract, 2019. 73(5): p. e13333. doi: 10.1111/ijcp.13333

36. Donald, B.J., S. Surani, H.S. Deol, U.J. Mbadugha, and G. Udeani, Spotlight on solithromycin in the treatment of community-acquired bacterial pneumonia: design, development, and potential place in therapy. Drug Des Devel Ther, 2017. 11: p. 3559-3566. doi: 10.2147/DDDT.S119545

37. Barrera, C.M., A. Mykietiuk, H. Metev, M.F. Nitu, N. Karimjee, P.A. Doreski, I. Mitha, C.M. Tanaseanu, J.M. Molina, Y. Antonovsky, et al., Efficacy and safety of oral solithromycin versus oral moxifloxacin for treatment of community-acquired bacterial pneumonia: a global, double-blind, multicentre, randomised, active-controlled, non-inferiority trial (SOLITAIRE-ORAL). Lancet Infect Dis, 2016. 16(4): p. 421-30. doi:

10.1016/S1473-3099(16)00017-7

38. File, T.M., Jr., B. Rewerska, V. Vucinic-Mihailovic, J.R.V. Gonong, A.F. Das, K. Keedy, D. Taylor, A. Sheets, P. Fernandes, D. Oldach, et al., SOLITAIRE-IV: A Randomized, Double-Blind, Multicenter Study Comparing the Efficacy and Safety of Intravenous-to-Oral Solithromycin to Intravenous-to-Oral Moxifloxacin for Treatment of Community-Acquired Bacterial Pneumonia. Clin Infect Dis, 2016. 63(8): p. 1007-1016. doi: 10.1093/cid/ciw490

39. Motsch, J., C. Murta de Oliveira, V. Stus, I. Koksal, O. Lyulko, H.W. Boucher, K.S. Kaye, T.M. File, M.L. Brown, I. Khan, et al., RESTORE-IMI 1: A Multicenter, Randomized, Double-blind Trial Comparing Efficacy and Safety of Imipenem/Relebactam vs Colistin Plus Imipenem in Patients With Imipenem-nonsusceptible Bacterial Infections. Clin Infect Dis, 2020. 70(9): p. 1799-1808. doi: 10.1093/cid/ciz530

40. Titov, I., R.G. Wunderink, A. Roquilly, D. Rodriguez Gonzalez, A. David-Wang, H.W. Boucher, K.S. Kaye, M.C. Losada, J. Du, R. Tipping, et al., A Randomized, Double-blind, Multicenter Trial Comparing Efficacy and Safety of Imipenem/Cilastatin/Relebactam Versus Piperacillin/Tazobactam in Adults With Hospital-acquired or Ventilator-associated Bacterial Pneumonia (RESTORE-IMI 2 Study). Clin Infect Dis, 2020. doi: 10.1093/cid/ciaa803

41. Metlay, J.P., G.W. Waterer, A.C. Long, A. Anzueto, J. Brozek, K. Crothers, L.A. Cooley, N.C. Dean, M.J. Fine, S.A. Flanders, et al., Diagnosis and Treatment of Adults with Community-acquired Pneumonia. An Official Clinical Practice Guideline of the American Thoracic Society and Infectious Diseases Society of America. Am J Respir Crit Care Med, 2019. 200(7): p. e45-e67. doi: 10.1164/rccm.201908-1581ST

42. Yang, J.W., L. Yang, R.G. Luo, and J.F. Xu, Corticosteroid administration for viral pneumonia: COVID-19 and beyond. Clin Microbiol Infect, 2020. 26(9): p. 1171-1177. doi: 10.1016/j.cmi.2020.06.020 (1) 438 ;

(1)

8

(1)

11

3

$s$
西 21

\section{(1)}

\section{4} 26 27

\section{8} 30

\section{2} 5 86 (1) 
43. Salluh, J.I.F., F.A. Bozza, M. Soares, J.C.R. Verdeal, H.C. Castro-Faria-Neto, E.S.J.R. Lapa, and P.T. Bozza, Adrenal response in severe community-acquired pneumonia: impact on outcomes and disease severity. Chest, 2008. 134(5): p. 947-954. doi: 10.1378/chest.08-1382

44. Abd-Elsalam, S., E.S. Esmail, M. Khalaf, E.F. Abdo, M.A. Medhat, M.S. Abd El Ghafar, O.A. Ahmed, S. Soliman, G.N. Serangawy, and M. Alboraie, Hydroxychloroquine in the Treatment of COVID-19: A Multicenter Randomized Controlled Study. Am J Trop Med Hyg, 2020. 103(4): p. 1635-1639. doi: 10.4269/ajtmh.20-0873

45. Dequin, P.F., N. Heming, F. Meziani, G. Plantefeve, G. Voiriot, J. Badie, B. Francois, C. Aubron, J.D. Ricard, S. Ehrmann, et al., Effect of Hydrocortisone on 21-Day Mortality or Respiratory Support Among Critically Ill Patients With COVID-19: A Randomized Clinical Trial. JAMA, 2020. 324(13): p. 1298-1306. doi: 10.1001/jama.2020.16761

46. Tomazini, B.M., I.S. Maia, A.B. Cavalcanti, O. Berwanger, R.G. Rosa, V.C. Veiga, A. Avezum, R.D. Lopes, F.R. Bueno, M. Silva, et al., Effect of Dexamethasone on Days Alive and Ventilator-Free in Patients With Moderate or Severe Acute Respiratory Distress Syndrome and COVID-19: The CoDEX Randomized Clinical Trial. JAMA, 2020. 324(13): p. 1307-1316. doi: 10.1001/jama.2020.17021

47. Edalatifard, M., M. Akhtari, M. Salehi, Z. Naderi, A. Jamshidi, S. Mostafaei, S.R. Najafizadeh, E. Farhadi, N. Jalili, M. Esfahani, et al., Intravenous methylprednisolone pulse as a treatment for hospitalised severe COVID-19 patients: results from a randomised controlled clinical trial. Eur Respir J, 2020. 56(6). doi: 10.1183/13993003.02808-2020

48. Li, N. and J. Hua, Interactions between mesenchymal stem cells and the immune system. Cell Mol Life Sci, 2017. 74(13): p. 2345-2360. doi: 10.1007/s00018-017-2473-5

49. Gupta, N., A. Krasnodembskaya, M. Kapetanaki, M. Mouded, X. Tan, V. Serikov, and M.A. Matthay, Mesenchymal stem cells enhance survival and bacterial clearance in murine Escherichia coli pneumonia. Thorax, 2012. 67(6): p. 533-9. doi: 10.1136/thoraxjnl-2011-201176

50. Dahl, S.L., J.S. Woodworth, C.J. Lerche, E.P. Cramer, P.R. Nielsen, C. Moser, A.R. Thomsen, N. Borregaard, and J.B. Cowland, Lipocalin-2 Functions as Inhibitor of Innate Resistance to Mycobacterium tuberculosis. Front Immunol, 2018. 9: p. 2717. doi: 10.3389/fimmu.2018.02717

51. Meng, F., R. Xu, S. Wang, Z. Xu, C. Zhang, Y. Li, T. Yang, L. Shi, J. Fu, T. Jiang, et al., Human umbilical cord-derived mesenchymal stem cell therapy in patients with COVID-19: a phase 1 clinical trial. Signal Transduct Target Ther, 2020. 5(1): p. 172. doi: 10.1038/s41392-020-00286-5

52. Shi, L., H. Huang, X. Lu, X. Yan, X. Jiang, R. Xu, S. Wang, C. Zhang, X. Yuan, Z. Xu, et al., Effect of human umbilical cord-derived mesenchymal stem cells on lung damage in severe COVID-19 patients: a randomized, double-blind, placebo-controlled phase 2 trial. Signal Transduct Target Ther, 2021. 6(1): p. 58. doi: 10.1038/s41392-021-00488-5

53. Shu, L., C. Niu, R. Li, T. Huang, Y. Wang, M. Huang, N. Ji, Y. Zheng, X. Chen, L. Shi, et al., Treatment of severe COVID-19 with human umbilical cord mesenchymal stem cells. Stem Cell Res Ther, 2020. 11(1): p. 361. doi: 10.1186/s13287-020-01875-5

54. Hashemian, S.R., R. Aliannejad, M. Zarrabi, M. Soleimani, M. Vosough, S.E. Hosseini, H. Hossieni, S.H. Keshel, Z. Naderpour, E. Hajizadeh-Saffar, et al., Mesenchymal stem cells derived from perinatal tissues for treatment of critically ill COVID-19-induced ARDS patients: a case series. Stem Cell Res Ther, 2021. 12(1): p. 91. doi: 10.1186/s13287-021-02165-4

55. Kong, D., X. Liu, X. Li, J. Hu, X. Li, J. Xiao, Y. Dai, M. He, X. Liu, Y. Jiang, et al., Mesenchymal stem cells significantly improved treatment effects of Linezolid on severe pneumonia in a rabbit model. Biosci Rep, 2019. 39(9). doi: 10.1042/BSR20182455 